Apidologie, 1977, 8 (4), 413-418.

\title{
REAKTION VON HONIGBIENEN AUF DIE HONIGTAUABGABE DER GRÜNEN TANNENHONIGLAUS BUCHNERIA PECTINATAE NÖRDL.
}

\author{
Réaction des abeilles à l'émission de miellat \\ par l'aphidien Buchneria pectinatae Nördl.
}

Joze RIHAR

Biotechnische Fakultät, Universität Ljubljana Ljubljana (Jugoslawien)

\begin{abstract}
SUMMARY
REACTION OF HONEYBEES ON THE EXCRETIONS OF THE APHID Buchneria pectinatae NördL. (Aphidina, Lachnidae)
\end{abstract}

Under laboratory conditions there were observed no changements in the rate of excretion of Buchneria pectinatae Nördl. In the field however the aphids excreted most during the morning and the evening, at noon-time the excretion was most reduced. The differences are highly significant. The collection of the honeydew by the honeybees has a very simular and corresponding course. The bees react on the concentration of the assimilates in the phloem sap of the firs which changes often during the vegetation period. Measurements in 1961 and 1962 demonstrate a negative correlation between concentration and atmospheric pressure (Rihar, 1963). Stained excretions (Meconium?) are most frequent during noon-time.

\section{ZUSAMMENFASSUNG}

Die Honigtau-Abgabe der grünen Tannen-Honiglaus Buchneria pectinatae Nördl. verläuft im Laboratorium gleichbleibend kontinuierlich. Im Freiland ist sie jedoch am stärksten am Morgen und am Abend, am schwächsten zur Mittagszeit. Die Unterschiede dabei sind hochsignifikant. Das Eintragen des Tannen-Honigtaues verläuft ähnlich. Die Bienen reagieren dabei auch auf die Konzentration der Tannen-Assimilate, welche sich im Verlaufe der Vegetationsperiode häufiger ändert. Messungen aus den Jahren 1961 und 1962 zeigen, daß sich die Konzentrationen in negativer Korrelation zum Atmosphärendruck hefinden (RIHAR, 1963). Gefärbte Exkrete (Meconium?) finden sich besonders häufig in den Mittagsstunden. 


\section{I. - EINLEITUNG}

Unser Bericht bezieht sich auf die Untersuchung der Grundlagen für die Prognose von Gradationen der grünen Tannen-Honiglaus Buchneria pectinatae Nördl., einer Art, welche in Slowenien die bedeutendste Quelle des Waldhonigs darstellt. Unser Ziel war es, entsprechende Methoden zu finden und zu entwikkeln. Hier sollen einige Beobachtungen betreffs der Unregelmässigkeiten in ihrer Exkretion während der Vegetationsperiode und während des Tages dargelegt werden. Von Interesse war die Frequenz, die Konzentration, das Aussehen des Exkretes sowie das Fluktuieren der Konzentration der Assimilate während der Saison.

\section{II. - MATERIAL UND METHODE}

Mit Fangplatten $(22,5 \times 25,5 \mathrm{~cm})$, die aus den zuerst gebrauchten gelb gefärbten Mitscherlich-Gefässen zum Fangen der Männchen der Aphide Buchneria pectinatae entstanden sind, wurde unter den Tannenbäumen die Frequenz der Honigtau-Abgabe ermittelt (Rinar, 1963, 1971). Die Platten wurden während mehrerer Jahre, in verschiedenen Jahreszeiten und verschiedenen geographischen Lagen und Meereshöhen nach Möglichkeit auf dieselbe Stelle gelegt und die Zahl, Grösse und Formen der Tröpfchen registriert.

Um Auskunft über die Exkretion unter Laborbedingungen zu bekommen, wurde ein Hygrothermograph umgebaut, so daß die Exkrete auf eine automatisch sich bewegende, mit Plastikfolie umgebene Walze fielen.

Die Exkrete wurden aus der Honigblase der heimkehrenden Bienen entnommen, mit Hilfe eines Handrefraktometers (Fabrikat Ballingham and Stanley) die Trockensubstanz (Konzentration) bestimmt. Um diese Methodik zu kontrollieren, wurde ein Vergleich zwischen der Konzentration des Honigtaues bei den Bienen, welche auf Tannennadeln gefangen wurden und solchen Bienen, die am Bienenstand zu Bestimmungszwecken genommen wurden, durchgeführt. Zwischen beiden Gruppen wurde kein signifikanter Unterschied gefunden.

\section{III. - ERGEBNISSE}

\section{Zusammenfassend werden folgende Resultate angegeben :}

1. Im Laboratorium haben Imago und Larven von $B$. pectinatae bei konstanter Temperatur $\left(20-21^{\circ} \mathrm{C}\right)$ während $30 \mathrm{bzw}$. 42 Stunden bei Tag und Nacht kontinuierlich Tröpfchen abgegeben. Die Larven haben ungefähr 2,7 mal mehr Exkrete als ihre Mütter ausgeschieden.

2. Die Exkretion in der Natur wurde morgens, mittags und abends auf 14. Platten registriert (Tab. 1).

Die Varianzanalyse hat gezeigt :

$A$ gegen $B C$ ist signifikant bei $0,0001(F=12,42 \times x \times)$

$B$ und $C$ sind signifikant bei $0,0005(F=16,50 \times x x)$

$A$ und $C$ sind nicht signifikant $(F=2,20)$ 
TAB. 1. - Exkretion unter Freilandverhältnissen zu verschiedenen Zeiten.

TABL. 1. - Excrétion à diffêrentes heures de la journée en conditions de champ.

\begin{tabular}{|c|c|c|c|}
\hline & \multicolumn{3}{|c|}{$\begin{array}{l}\text { Zeit derBeobachtung } \\
\text { Moment de l'observation }\end{array}$} \\
\hline & $6.30 \stackrel{\mathrm{A}}{=} 7.30$ & $12.30 \stackrel{B}{-} 13.30$ & $17.30 \stackrel{\mathrm{C}}{-} 18.30$ \\
\hline $\begin{array}{l}\text { Min. und max. Zahl der Exkrettropfen } \\
\text { auf einer Platte } \ldots \ldots \ldots \ldots \ldots \ldots \ldots \ldots \\
\text { Nombre min. et max. de gouttes émises sur une } \\
\text { lame }\end{array}$ & $6-83$ & $7-32$ & $18-81$ \\
\hline $\begin{array}{l}\text { Gesamtzahl auf } 14 \text { Platten } \ldots \ldots \ldots \ldots \ldots \\
\text { Nombre total sur } 14 \text { lames }\end{array}$ & 704 & 248 & 534 \\
\hline $\begin{array}{l}\text { Durchschnittszahl } \ldots \ldots \ldots \ldots \ldots \ldots \ldots \\
\text { Moyenne }\end{array}$ & 50 & 18 & 38 \\
\hline
\end{tabular}

3. Zum Sammeln des Honigtaues durch die Bienen : Am 20.7.1960 wurden die Waagstockergebnisse jede Stunde, am 23.6.1962 jede zweite Stunde registriert. Beide Kurven, die aus Graphiken ersichtlich sind, zeigen das erste Maximum des Waagstockzuwachses ungefähr um 7-11 Uhr vormittags und das zweite nach 16.00 Uhr nachmittags.

4. Zur Frage der Konzentration der Assimilate während der Vegetationsperiode werden zwei Beobachtungen angegeben :

a) Die Konzentration der Assimilate während der Saison verändert sich. Vom 30. Juli $(n=15)$ bis zum 8 . August $(n=8)$, gemessen in beiden Fällen um 9.00 Uhr vormittags, ist sie z.B. von durchschnittlich 25,10\% auf durchschnittlich 39,00\% gestiegen. Die Zunahme der Konzentration haben wir im Laufe der Saison auch beim Nektar von Satureia subspicata festgestellt.

b) Über die Abhängigkeit der Konzentration der Assimilate vom Barometerdruck haben wir in Prag berichtet (Rinar, 1963). Wir haben bewiesen, dass sie in negativer Korrelation zum Atmosphärendruck steht. Das zeigen folgende Regressionslinien :

für Hrusica $1961(y=25,51-0,127 x ; r=0,592)$

für Rakitna $1962(y=44,24-0,448 x ; r=0,697)$

5. Die Konzentration der Exkrete ist während des Tages nicht gleich. Im Jahre 1964, wurden folgende Werte gefunden (Tab. 2).

Allem Anschein nach ist der Honigtau in den Morgenstunden dicker als zu Mittag, abends konzentriert er sich erneut. 
TAB. 2. - Konzentration der Exkrete zu verschiedenen Daten und Tageszeiten.

TABL. 2. - Concentration des excrétats à différentes dates et heures.

\begin{tabular}{|c|c|c|c|c|c|}
\hline & \multicolumn{5}{|c|}{$\begin{array}{l}\text { Zeit der Beobachtung } \\
\text { Moment de l'observation }\end{array}$} \\
\hline & $\begin{array}{l}\text { Am } 6.8 \\
\text { le } \\
\text { um } 6.30 \\
\text { à }\end{array}$ & $\begin{array}{l}\text { Am } 8.8 \\
\text { um } 9.00 \\
\text { à }\end{array}$ & $\begin{array}{l}\text { Am } 6.8 \\
\text { le } \\
\text { um } 13.00 \\
\text { à }\end{array}$ & $\begin{array}{l}\text { Am } 7.8 \\
\text { le } \\
\text { um } 18.00 \\
\text { à }\end{array}$ & $\begin{array}{l}\text { Am } 5.8 \\
\text { le } \\
\text { um } 19.00 \mathrm{Uhr} \\
\text { à }\end{array}$ \\
\hline $\begin{array}{l}\text { Zahl der Messungen } \ldots \\
\text { Nombre de mesures }\end{array}$ & 11 & 8 & 12 & 5 & 10 \\
\hline $\begin{array}{l}\text { Min. Max. Konzentration. } \\
\text { Concentrations min. et max. }\end{array}$ & $24-48,50$ & $27-46$ & $24,50-43$ & $23-4.3$ & $25,50-49$ \\
\hline $\begin{array}{l}\varnothing \text { Konzentration } . . . \ldots \\
\text { Concentration moyenne }\end{array}$ & 37,55 & 39,00 & 34,72 & 32,30 & 37,80 \\
\hline
\end{tabular}

6. Als Nebenresultat möchten wir das Auftreten gefärbter, nicht determinierter Exkrete erwähnen. Unter den gewöhnlich durchsichtigen Tröpfchen findet man sehr selten auch gefärbte, flache.

In bestimmten Jahren und Lagen sind sie seltener, in anderen häufiger. Im Jahre 1964, als von wässrigem Honigtau gesprochen wurde, haben wir unter 1586 Exkret-Tropfen 15, d.h. 0,96\% gefärbte gefunden. Den Tageszeiten nach waren sie auf folgende Weise verteilt (Tab. 3).

TAB. 3. - Auftreten gefürbter Exkrettropfen.

TaBL. 3. - Existence de gouttes d'excrétat colorées.

\begin{tabular}{|c|c|c|}
\hline & & $\begin{array}{l}\text { gefärbte Exkrete } \\
\text { Excrétats colorés }\end{array}$ \\
\hline $\begin{array}{l}\text { Uhrzeit } \\
\text { Heure }\end{array}$ & $\mathbf{n}$ & $\begin{array}{c}\text { In } \% \text { aller Exkrete } \\
\text { En } \% \text { des excrétats totaux }\end{array}$ \\
\hline $6,30-7,30$ & 2 & 0,24 \\
\hline $12,30 \ldots 13,30$ & 11 & 4,44 \\
\hline $117,30-18,30$ & 2 & 0,96 \\
\hline
\end{tabular}


Man kann diese Exkrete, welche vermutlich das Meconium der Aphiden darstellen, als rötlich, dunkelrot bis schwarz, seltener auch milchig, dem $\mathrm{Ge}$ schmack nach als nicht süss bezeichnen.

\section{IV. - DISKUSSION}

Aus den dargelegten Daten ist ersichtlich, daß Frequenz und Konzentration der Exkrete der grünen Tannen-Honiglaus Buchneria pectinatae mancherlei Einflüssen unterworfen sind. Auf die gleichen Einflüsse reagieren die Bienen beim Sammeln des Honigtaues.

Die Haupttracht auf der Tanne ist nach Matko (1917) zwischen 7 und 10 Uhr morgens und nachmittags von 15 Uhr bis zur späten Dämmerung. Nach Michel (1942) gibt es bei der Exkretion keine Regelmäßigkeit, nach LeonhardT (1940) gibt es ein Maximum um Mitternacht und ein ausgeprägtes Minimum um $10 \mathrm{Uhr}$ vormittags. Aus unseren Messungen ist eine Parallelität zwischen der Intensität des Sammelns durch die Bienen während des Tages und der Frequenz in der Exkretion der grünen Aphide morgens, mittags und abends ersichtlich.

Im Gegensatz zu der beschriebenen Rhythmik steht die Frequenz der gefärbten, noch nicht genauer geklärten Exkrete (Meconium ?). Diese selten gefundenen Exkrete sind am häufigsten in der Zeit des schwächsten HonigtauEintrages.

Sowohl durch die genannten Einflüsse als auch durch das Fluktuieren der Konzentration des Tannenhonigtaues in der Vegetationsperiode wird die Zuverlässigkeit der kurzfristigen Prognose beeinträchtigt. Man muß eben voraussehen können, wie die Bienen reagieren werden, wenn der Barometerdruck steigt oder fällt und wenn die Konzentration der Assimilate ansteigt oder abfällt. (Es geht hier um zwei Erscheinungen, die von einander unabhängig sind). Nur bei idealen Laboratoriumsbedingungen, bei denen wir ähnliche Befunde gewonnen haben wie früher Mitrler (1958), könnte man hundertprozentig Trachtenaussichten voraussagen. Schließlich muß betont werden, daß man bei der Honigtauprognose ausser den Hauptparametern (RIHAR, 1971) noch mehrere Nebenfaktoren in Betracht ziehen muß, um bei seiner Arbeit sicherer zou sein.

Eingegangen im Juni 1977.

Reçu pour publication en juin 1977.

\section{RÉSUMÉ}

L'émission de miellat par Buchneria pectinatae Nördl. en laboratoire a lieu de façon uniformément continue. En champ par contre elle marque un maximum le matin et le soir et un minimum à midi. Les différences sont hautement significatives. La récolte de miellat par les 
abeilles ne varie pas dans ses modalités. Les abeilles réagissent à la concentration des assimilats de la sève élaborée du sapin, qui varie souvent au cours de la période de végétation. Les mesures effectuées en 1961 et 1962 révèlent une corrélation négative entre la concentration et la pression atmosphérique (RıHAR, 1963). Des excrétions colorées (Meconium?) sont plus fréquentes dans le milieu de la journée.

\section{LITERAT UR}

LeonhardT H., 1940. - Beiträge zur Kenntnis der Lachniden, der wichtig sten Tannenhonigtauerzeuger. $Z$. ang. Ent., 27, 208-281.

Matкo M., 1917. - Kako sem pridelal letos iz enega AŽ panja $80 \mathrm{~kg}$ medu. Slov. cebelar, 20, 118-120.

Mrchel E., 1942. - Beiträge zur Kenntnis von Lachnus (Pterochlorus) roboris L., einer wichtigen Honigtauerzeugerin an der Eiche. Z. ang. Ent., 2, 243-281.

MittLer T. E., 1958. - Studies on the feeding and nutrition of Tuberolachnus salignus (Gmelin) (Homoptera, Aphididae), J. exper. Biologie, 35, 626-638.

Rifar J., 1963. - Einfluss der äusseren Faktoren auf die Konzentration des Tannenhonigtaues. XIX. Apimondia Kongress, Prag, 648-651.

Rimar J., 1964. - Periodicnost pojave mane zelene jeline vasi (Buchneria pectinatae Nördl.) u SR Sloveniji i njen uticaj na razvoj pcelinjih drustava. Doktorska dizertacija. Ljubljana, $1-203$.

Rihar J., 1971. - Methoden der Prognosierung des Honigtaues auf der Tanne. XXII. Apimondia Kongress, Moskau, 590-592. 